\title{
Utilización de Mapas de Conocimiento Difuso (MCD) en la asignación de prioridades de la restauración fluvial: Aplicación al río Esla
}

\author{
Solana Gutiérrez, J., Rincón Sanz, G., Alonso González, C., García de Jalón \\ Lastra, D. \\ ${ }^{1}$ ETSI Montes, Forestal y del Medio Natural, Universidad Politécnica de Madrid. Av. Ramiro de Maeztu s/n. Ciudad \\ Universitaria. 28040 Madrid (España \\ *e-mail: joaquin.solana@upm.es
}

\section{Resumen}

La gestión de los ecosistemas fluviales atañe a diferentes grupos de interés (i.e., gestores, profesionales, académicos y usuarios) que tienen concepciones y objetivos diferentes en relación al mismo. Diversos procedimientos se han ideado para permitir la participación de estos actores en la planificación hidrológica; si bien los procedimientos con puesta en común de intereses y responsabilidad compartida en la gestión se muestran más sostenibles ecológica y temporalmente. La modelización matemático-física de los sistemas fluviales es muy aconsejable en el planeamiento para poder plantear escenarios y predecir soluciones de consenso, pero no siempre se tiene información suficientemente para realizarla. En estos casos, los modelos semánticos o meta-modelos pueden ser alternativas válidas para simular estos sistemas complejos multi-criterio con múltiples decisores. En particular, los Mapas Cognitivos Difusos (MCD) se presentan como una herramienta que facilita la modelización de los sistemas ecológicos, sus funciones y servicios. Los MCD son redes de conceptos entrelazados mediante relaciones causales. La ordenación espacial de los conceptos y la aplicación de reglas de lógica difusa facilita la integración de MCD generados por diferentes expertos, produciendo mapas de consenso. Una vez establecida la matriz de adyacencia del MCD, se somete al modelo a distintos escenarios de actuación simulándose los efectos en las funciones y servicios ambientales.

En el presente trabajo se aplican los MCD en el estudio de alternativas de restauración fluvial del río Esla, donde participan 7 decisores pertenecientes a 3 grupos de expertos. Como resultado de la modelización del río Esla se obtiene una previsión de los efectos esperables en el ecosistema fluvial ante distintos niveles de actuación restauradora.

Palabras clave: conocimiento experto, mapas cognitivos difusos, meta-modelos, modelización de ecosistemas fluviales 


\section{Introducción}

La presión humana sobre los ecosistemas fluviales ha ido en aumento a lo largo del tiempo forzando su capacidad ecológica y su resiliencia, actualmente la mayoría de los ecosistemas fluviales se encuentran fuertemente modificados y muy limitada su capacidad ecológica (Kauffman et al., 1997). La gestión de los ecosistemas fluviales en climas mediterráneos trata de regular uno de los ecosistemas más complejos donde el interés social por los recursos hídricos choca con la calidad, fragilidad e importancia ecológica del ecosistema fluvial.

La continua intervención humana en los ríos ha provocado la degradación, cuando no la pérdida, de las funciones y servicios ambientales que ofrecen los ecosistemas fluviales, afectando negativamente al hombre y a su cultura (González del Tánago et al., 1995). Esta doble pérdida de los valores, ambientales y antrópicos, ha generado una respuesta social en forma de normativas, reglamentos y leyes cuyo fin es la recuperación de la calidad ecológica de los ríos y sus entorno (CEE, 2000). Para lograr la recuperación de la calidad ecológica y su mantenimiento sostenible es necesario establecer planes de gestión que integren la naturaleza multifactorial del sistema fluvial, apliquen metodologías multi-criterio y multi-objetivo y permitan una efectiva participación pública.

Con el fin de explorar nuevas metodologías que permitan una efectiva participación pública en la elaboración de los planes de gestión, se propone el uso de Mapas Cognitivos Difusos (MCD). Los MCD facilitan la visualización de una red de conceptos y factores sistémicos que se vinculan mediante relaciones causa-efecto de naturaleza semántica (Papageorgiou et al., 2009). Una vez generado un MCD, se puede someter al sistema a diferentes escenarios de entrada y obtener las respuestas más verosímiles en sus componentes, funciones y productos. El establecimiento de una multiplicidad de posibles escenarios a los que se puede enfrentar en el futuro el sistema es preferible a una única predicción óptima (Kok, 2009).

En definitiva, el presente artículo propone:

1. Valorar el potencial metodológico de los MCD en la gestión de sistemas fluviales.

2. Estudiar el alcance del criterio experto y la participación pública bajo lógica difusa en la toma de decisiones ambientales.

3. Simular el comportamiento del sistema ante posibles escenarios.

\section{Material y métodos}

\subsection{Mapas cognitivos difusos}

Los Mapas Cognitivos Difusos (MCD) constituyen una atractiva técnica de modelado para los sistemas complejos (Papageorgiou et al., 2009). Mediante los MCD se pueden describir los principales aspectos del comportamiento de sistemas en términos de conceptos y relaciones causales (Papageorgiou and Kontogianni, 2012). 
Los MCD son meta-modelos semánticos que describen el flujo de operación de un sistema dado (Özesmy and Özesmi, 2004). Los mapas de conocimiento fueron primeramente utilizados por Axelrod (1976) para modelar el comportamiento de grupos políticos, pero fue Kosko (1986) quien incorporó el enfoque no determinístico o "difuso" a las relaciones causales entre conceptos.

Los MCD se modelan mediante un grafo orientado formado por "nodos" (i.e., conceptos) y "líneas" relativas a las relaciones causales entre los conceptos. El modelo matemático del MCD se compone de un vector de variables de estado (conceptos), con valores en el dominio [0,1], y una matriz de adyacencia que simula las uniones causa-efecto entre conceptos, formada por coeficientes en el intervalo $[-1,1]$ (Banini and Bearman, 1998). Con estos dos objetos se define un proceso de Markov donde se fuerza la convergencia mediante un filtro logístico (o semántico) en cada paso.

Para construir un MCD cada experto, primeramente, refleja "su conocimiento" del sistema en un conjunto de conceptos (variables, nodos o componentes) y, posteriormente, gradúa semánticamente las relaciones causa-efecto entre conceptos mediante reglas del tipo "si-entonces". El resultado es la asignación de un calificativo lingüístico (i.e., muy alta, alta, media, baja, muy baja) para cada conexión entre nodos (Stylos and Groumpos, 2000, 2004; Papageorgiou and Groumpos, 2005). Aunque esta información es deseable que sea aportada directamente por el experto mediante un dibujo de una red conceptual, también puede obtenerse por medio de cuestionarios o entrevistas mediante encuestadores (Özesmy and Özesmy, 2004). Posteriormente, las calificaciones semánticas son transformadas en valores en el intervalo $[-1,1]$ que reflejan una relación de orden.

En resumen, los MCD son un conjunto estructurado de los siguientes elementos (Kok, 2009):

- Los conceptos $\left(C_{1}, C_{2}, \ldots, C_{n}\right)$, que describen las principales características del sistema.

- El vector inicial $\left(A_{1}, A_{2}, \ldots, A_{n}\right)$, que representa el estado de los conceptos.

- Los arcos, $\left(\bar{C}_{1} C_{2}, \bar{C}_{2} C_{3}, \ldots, C_{k} C_{k+1}\right)$, que establecen las relaciones (positivas o negativas) entre conceptos.

- La matriz de adyacencia, $\left(E_{c}\right)$, que contiene todos los valores de las relaciones entre conceptos (valores entre -1 y 1 ).

Una vez realizadas las entrevistas al panel de expertos, se obtendrá un MCD por cada participante que se transformará en una matriz de adyacencia (Harary et al., 1965). Los mapas procedentes de distintos participantes se fusionarán en aquellos conceptos análogos o similares. Los conceptos no asimilables se añadirán generando nuevos mapas integrados de percepción del sistema. La combinación de matrices se realizará normalizando cada elemento por el número de expertos según la ecuación (Banini and Bearman, 1998): $E_{c}=\sum_{i=1}^{k} E_{i} / k$ donde $E_{c}$ es la matriz de adyacencia combinada, $k$ es el número de expertos y $E_{i}$ son las matrices de adyacencia de los distintos expertos $i$.

El valor de entrada del proceso markoviano va transformándose en cada iteración (i.e., $A_{j 1, \ldots}, A_{j, t+1,} A_{j, t+\mathrm{m}}$ ) hasta convergencia en un estado estable. Debido al carác- 
ter iterativo de este procedimiento, los estados del MCD evolucionan a lo largo del tiempo hacia nuevos estados de equilibrio (Curia and Lavalle, 2011). Después de cada iteración, los valores del vector resultante se transforma en una variable de estado binaria [1/0] mediante una función de filtro logística, $f(x)=1 /\left(1+e^{-\mathrm{mx}}\right)$, donde es un número real positivo y $x$ es el valor de vector en el punto de equilibrio. Ésta función permite asignar y expresar semánticamente un estado categórico (o binario) a cada componente en cada iteración.

Los MCD están compuestos por gran número de variables componentes e interconexiones. Las variables se clasifican según su influencia en el resto de componentes según: variables transmisoras (denominadas: fuerzas, drivers), variables receptoras (denominadas: estados respuestas, productos finales) y variables ordinarias (denominadas: medios, factores) (Bougon et al., 1977; Eden et al., 1992; Harary et al., 1965)

La teoría de grafos proporciona una forma de analizar la estructura de los MCD (Özesmy and Özesmy, 2003) mediante tres indicadores: indicador "outdegree", indicador "indegree" e indicador de "centralidad". El indicador outdegree es la suma de los valores de la matriz de adyacencia asociados con los conectores que salen de un nodo o variable; una variable transmisora presenta un alto outdegree. El indicador indegree es la suma de los valores de la matriz de adyacencia asociados a las conexiones que entran en un nodo y muestra el grado de dependencia de la variable; una variable receptora presenta un alto indegree. La centralidad es la suma de los indicadores outdegree y el indegree, e indica el grado de participación o importancia de la variable en el sistema (Özesmy and Özesmy, 2003, 2004).

\subsection{Zona de estudio y toma de datos}

El río Esla es un afluente del río Duero por la margen derecha. Tiene una longitud de $287.83 \mathrm{~km}$ y una cuenca de $16026 \mathrm{~km}^{2}$. Nace en la provincia de León y desemboca en el Duero en la provincia de Zamora. Tiene una aportación media de 5066 $\mathrm{hm}^{3}$ al año (CHD, 2014).

En el recorrido del río Esla se encuentran dos grandes embalses; primero, el embalse de Riaño que está situado en la cabecera con una capacidad máxima de 650 $\mathrm{hm}^{3}$ y una superficie de $817.51 \mathrm{~km}^{2}$, segundo, el embalse de Ricobayo, situado cerca de la desembocadura, con una capacidad máxima de $1179 \mathrm{hm}^{3}$ y que ocupa una superficie de $159.52 \mathrm{~km}^{2}$. Ambos embalses condicionan el régimen natural de caudales, produciéndose una inversión del mismo al haber mayor disponibilidad de agua en los meses de verano, coincidentes con la campaña agrícola de regadío, que en la época invernal de máxima pluviosidad. También se hallan en la cuenca los canales Alto de Payuelos, Cea-Carrión, Curueño-Porma y Páramo Bajo que juegan un papel importante en la regulación de la cuenca del Esla (CHD, 2014).

Este sistema fluvial atiende el abastecimiento de 278000 personas (incluyendo la ciudad de León), el regadío de 97000 ha, el suministro de tres piscifactorías y la refrigeración de las centrales térmicas de producción de energía de la Robla. Además, sus aguas se turbinan en 19 centrales hidroeléctricas con una potencia conjun- 
ta de $451 \mathrm{MW}$ y una producción media de $1115 \mathrm{GWh}$. Se prevé un notable aumento de la superficie a regar a partir del año 2015 (CHD, 2014).

Se han realizado 7 entrevistas a 2 técnicos gestores de cuenca, 2 académicos y 3 usuarios conocedores del río Esla y su entorno. Al comienzo de las entrevistas, cada participante recibió una plantilla con una serie de variables ó componentes sistémicas predefinidas por los autores, de manera que sirvieran de guía orientativa (Tab. 1). También se les proporcionó una tabla de valoración semántica (Tab. 2). La graduación semántica de las conexiones se fijaron respondiendo a la pregunta: “¿En qué medida piensa usted que la variable $\mathrm{X}$ influye (signo +) o es influida (signo -) por otra(s) variable(s)?" (Mouratioadou and Moran, 2007).

Tabla 1. Conceptos componentes del MCD.

\begin{tabular}{|l|l|}
\hline \multicolumn{1}{|c|}{ Variable } & \multicolumn{1}{c|}{ Definición } \\
\hline Barreras, embalses y azudes & $\begin{array}{l}\text { Conjunto de barreras artificiales } \\
\text { que obstruyen el flujo natural de las aguas }\end{array}$ \\
\hline Régimen hidrológico natural & Flujo del agua en condiciones naturales \\
\hline Calidad de las aguas & $\begin{array}{l}\text { Estatus físico-químico de las aguas } \\
\text { superficiales y subterráneas }\end{array}$ \\
\hline Dinámica de sedimentos & Balance erosivo, de transporte y sedimentación \\
\hline Producción agro-forestal & $\begin{array}{l}\text { Producción agrícola, forestal } \\
\text { y ganadera en los corredores fluviales }\end{array}$ \\
\hline Usos urbanos e infraestructuras & $\begin{array}{l}\text { Estructuras antrópicas que alteran } \\
\text { la dinámica natural de las aguas }\end{array}$ \\
\hline $\begin{array}{l}\text { Continuidad } \\
\text { y ancho de los paisajes riparios }\end{array}$ & Continuidad y anchura de la vegetación de ribera \\
\hline Aspectos socio-económicos & Relaciones entre el ecosistema fluvial y el hombre \\
\hline Vegetación riparia & $\begin{array}{l}\text { Especies y formaciones vegetales asociadas } \\
\text { con los ecosistemas riparios }\end{array}$ \\
\hline Comunidades fluviales & Poblaciones piscícolas y fluviales \\
\hline Producción hidroeléctrica & $\begin{array}{l}\text { Alteración de los ecosistemas fluviales } \\
\text { debido a la existencia de centrales hidroeléctricas. }\end{array}$ \\
\hline $\begin{array}{l}\text { Conectividad fluvial } \\
\text { (longitudinal, lateral y vertical) }\end{array}$ & Conectividad de los ecosistemas riparios \\
\hline Condiciones de las márgenes fluviales & Alteración de las márgenes fluviales \\
\hline
\end{tabular}

Una vez obtenidos los mapas individuales se combinaron en un único MCD colectivo, asignándose en cada concepto el promedio las matrices individuales de adyacencia $\left(E_{c}\right)$. Para facilitar la obtención automática de los indicadores outdegree, indegree y de centralidad del sistema se utilizó el software FCMapper FCM (Bachhofer and Wildenberg, 2010). También se utilizó esta aplicación informática para generar las simulaciones de los diferentes escenarios. 
Tabla 2. Interpretación de las relaciones causa-efecto

\begin{tabular}{|c|c|c|}
\hline Orden & Relación semántica & Valor \\
\hline-5 & Muy fuerte negativa & -1 \\
\hline-4 & Fuerte negativa & -0.8 \\
\hline-3 & Media negativa & -0.6 \\
\hline-2 & Débil negativa & -0.4 \\
\hline-1 & Muy débil negativa & -0.2 \\
\hline 1 & Muy débil positiva & 0.2 \\
\hline 2 & Débil positiva & 0.4 \\
\hline 3 & Media positiva & 0.6 \\
\hline 4 & Fuerte positiva & 0.8 \\
\hline 5 & Muy fuerte positiva & 1 \\
\hline
\end{tabular}

Las variables con las que se ha confeccionado el MCD colectivo son todas de tipo ordinario, lo que significa que existen relaciones causales entre ellas.

Las variables más importantes del MCD son aquellas que tienen altos valores de centralidad (Fig. 1), pues regulan la dinámica del sistema (Özesmi and Özesmi, 2004). La diferencia entre el indegree y el outdegree de una variable indican el tipo de variable: las variables que tienen altos valores outdegree influyen fuertemente en el resto de conceptos y son consideradas como fuerzas (e.g., producción agroforestal, usos urbanos, embalses) o presiones (e.g., alteración del régimen natural de cau-

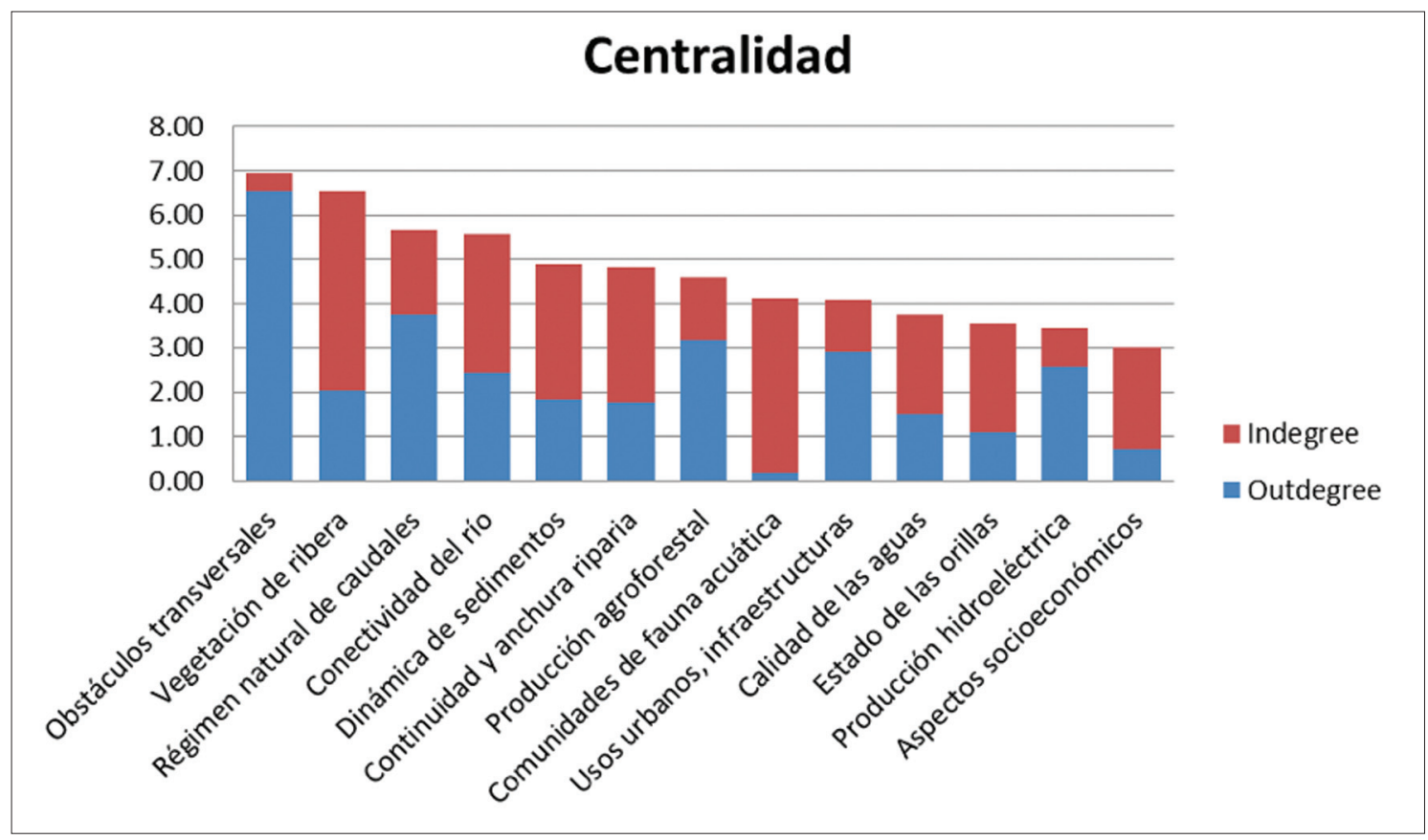

Figura 1. Indicadores de las variables componentes del MCD: Indicadores de "Centralidad" como suma de los indicadores “outdegree" (en azul) e "indegree” (en rojo) de las principales variables del MCD del río Esla. 
dales) del sistema de acuerdo con la denotación medioambiental FPEIR (i.e., Fuerzas, Presiones, Estado, Impacto, Respuesta) (OCDE, 1991); las variables que tienen altos valores de indegree se consideran variables de estado (e.g., conectividad, continuidad y anchura del corredor fluvial, vegetación de ribera) y son las que determinan el estatus ecológico del ecosistema (Gurnell et al., 2014).

Una vez determinado el papel que juega cada variable en el sistema, se establece un panel de escenarios para las variables fuerza del sistema y se obtiene un conjunto de resultados de las variables de estado (Fig. 2). Generalmente se parte de un vector inicial A0 donde las variables fuerza tienen valor 1 y se va rebajando el vector según deciles (e.i., 0.9, 0.8, 0.7, ..). Como ejemplo de este tipo de análisis se presentan dos simulaciones: primero, la simulación de la degradación continua del régimen natural de las aguas; segundo, la simulación de la eliminación paulatina de las barreras transversales del río.

\section{Resultados}

\subsection{Modelización del MCD}

En la primera fase de la modelización MCD del río Esla se obtuvieron siete mapas con $13.6 \pm 2.14$ (s.d.) nodos y $46.28 \pm 26.72$ (s.d.) conexiones. Los siete mapas se integraron en un MCD colectivo con 13 nodos y 114 conexiones (Tab. 3). Las variables con mayor "centralidad", "indegree" y "outdegree" se muestran en la Tabla 4.

Tabla 3. - Comparación de los valores individuales y colectivos del MCD

\begin{tabular}{|c|c|c|}
\hline Indicadores & Individuales & Colectivos \\
\hline Número de mapas & 7 & 1 \\
\hline Variables & $13.6 \pm 2.14$ & 13 \\
\hline Número de conexiones & $21.4 \pm 1.99$ & 114 \\
\hline Conexiones / variables & 1.58 & 8.77 \\
\hline Densidad & 0.124 & 0.67 \\
\hline
\end{tabular}

Tabla 4. - Variables con alta centralidad, indegree y outdegree.

\begin{tabular}{|c|c|c|}
\hline Centralidad & Indegree & Outdegree \\
\hline Barreras, embalses y azudes & Vegetación riparia & Barreras, embalses y azudes \\
\hline Vegetación riparia & Comunidades fluviales & Régimen hidrológico natural \\
\hline Régimen hidrológico natural & Conectividad fluvial & Producción agro-forestal \\
\hline
\end{tabular}

La variable con mayor centralidad fue "Barreras, presas y azudes", que influía fuertemente en muchas variables (Outdegree $=6.54$ ) y era escasamente influida por 
otras variables (Indegree $=0.4$ ). La variable de menor centralidad fue "Aspectos socioeconómicos", si bien presentó un remarcable indegree pero un pequeño outdegree. Las principales variables ordenadas según el indicador de centralidad se muestran en la Fig. 1.

Las variables que ejercieron más influencia (i.e., "fuerzas") en el resto de variables (Outdegree > Indegree) fueron: "Barreras, presas y azudes", "Régimen de flujo natural del agua", "Producción agro-forestal", "Usos urbanos" y "Producción hidroeléctrica". Por otra parte, "Vegetación riparia","Comunidades riparias", "Conectividad fluvial" y "Dinámica de sedimentos" fueron las componentes del sistema con mayor indegree (Indegree > Outdegree), calificándose como variables "estado" según FPEIR (Tab. 4).

En conjunto, el modelo MCD del río Esla muestra un sistema muy influenciado por la actividad humana, donde las variables económicas, relativas a producciones y a infraestructuras, presentan elevados valores de outdegree. Sin embargo, las componentes ambientales, esenciales para el buen funcionamiento del ecosistema, presentan un fuerte indegree, salvo el "régimen natural de caudales".

En la cuenca del río Esla no es sorprendente que la variable "Barreras, presas y azudes" sea la variable con mayor centralidad, pues es un elemento artificial que altera muchas otras componentes fluviales además de producir una fuerte alteración ecológica y respuesta social. También la "vegetación de ribera" y la "alteración del régimen natural" de las masas de aguas son variables muy afectadas por las actividades antrópicas.

Como se observa en la Fig. 1, las variables socioeconómicas tienen mayores valores indegree que la mayoría de las variables ambientales. El río Esla, como la mayoría de los ríos europeos, es un sistema degradado por las actividades humanas y, por lo tanto, es lógico que las variables socioeconómicas tengan un alto valor indegree consecuencia del efecto de multitud de presiones y fuerzas degradantes. Por otra parte, el "régimen hidrológico natural" de las aguas muestra un alto valor de centralidad debido a su alto outdegree condicionando a otras componentes de importancia (i.e., "barreras, azudes y presas" o la "producción agroforestal").

\subsection{Simulación de dos políticas de restauración fluvial}

Una vez determinado el MCD del río Esla, se puede simular la variación esperable de las componentes de estado ante distintos vectores de partida. Así, una disminución de la variable de estado en comparación con las condiciones de referencia indica un deterioro medioambiental, mientras que un valor positivo refleja una mejora en el ecosistema (Tan and Özesmi, 2006).

Identificadas los principales componentes e etiquetadas como "fuerzas" productivas y variables de "estado" en el MCD del Esla, se realizaron dos simulaciones de políticas de restauración fluvial: primero, una simulación de no intervención ante las fuerzas y presiones antrópicas, donde se produce un deterioro del régimen natural de las aguas y, segundo, una simulación de política ambientalmente intervencionista con eliminación de las barreras transversales existentes en el río Esla. 


\subsubsection{Simulación del deterioro del régimen natural de las aguas}

Se estableció un patrón de deterioro del "régimen hidrológico natural" según deciles respecto a la situación inicial (Fig. 2). Para cada decil se obtuvo un valor estable de las componentes calificadas como variables de estado: "continuidad riparia", "vegetación fluvial", "comunidades fluviales" y "conectividad fluvial".

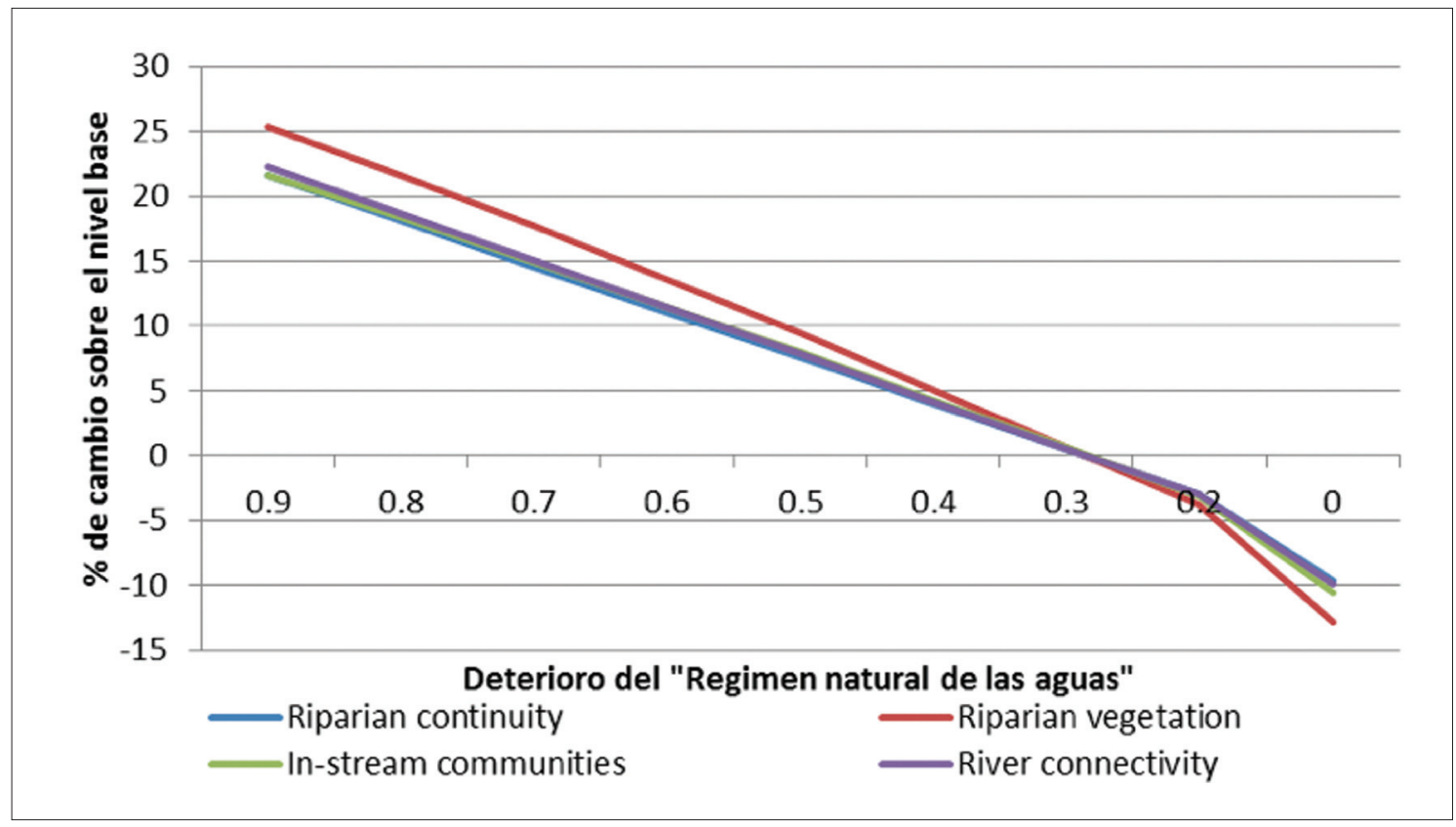

Figura 2. Simulación del efecto del deterioro del "Régimen natural de las aguas" (4a. Figura superior) y de la disminución de las "Barreras transversales" (4b. Figura inferior) en las variables de estado "Continuidad riparia" (en azul), "Comunidades fluviales" (en verde), "Vegetación riparia"(en rojo) y "Conectividad fluvial" (en violeta).

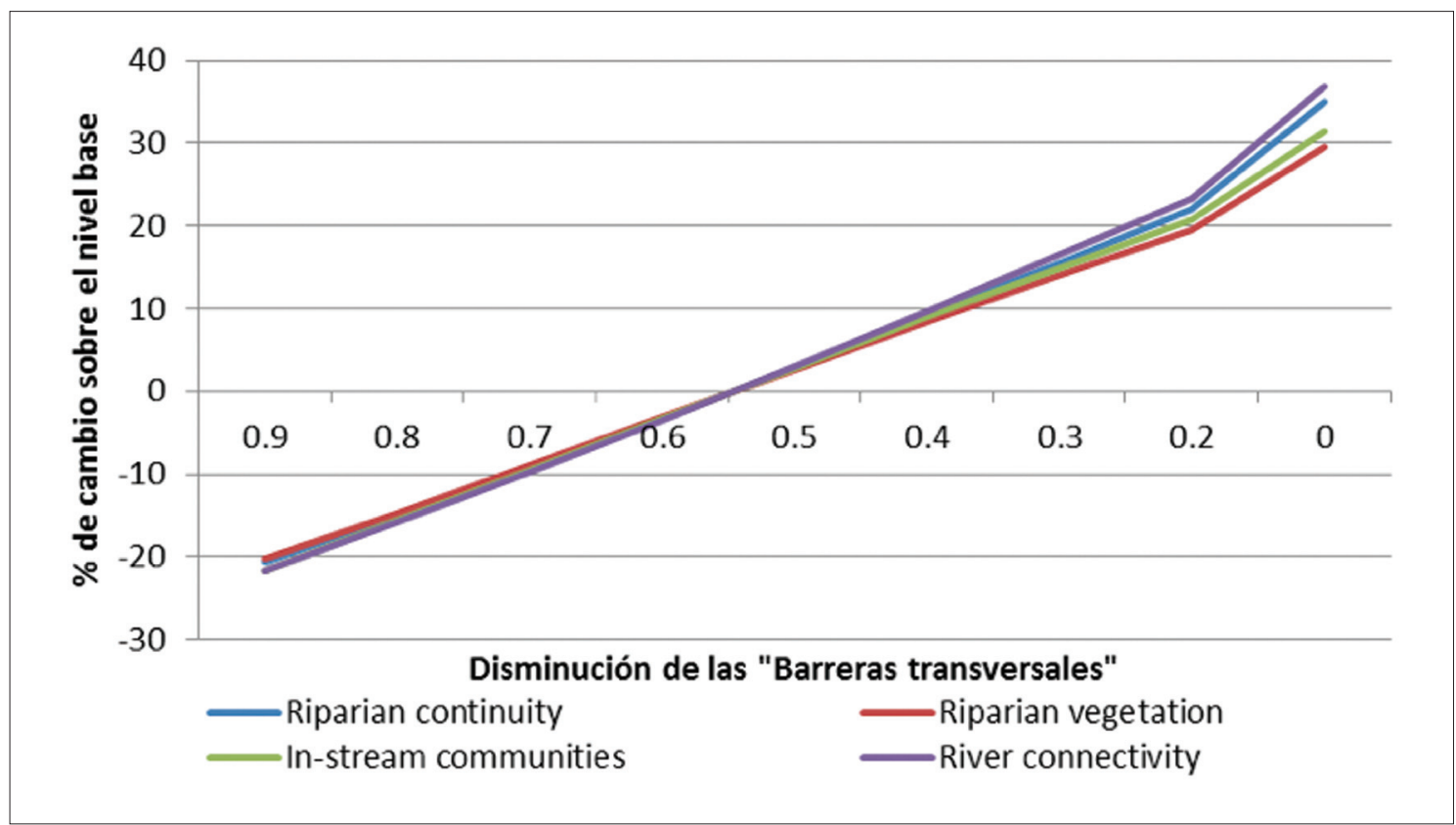


Una vez realizada la batería de simulaciones, se observó que el deterioro del "régimen hidrológico natural" generó un mayor deterioro en la "vegetación riparia" que en el resto de variables respuesta: "conectividad fluvial", "continuidad riparia" y "comunidades fluviales". La alteración se mantuvo lineal para deterioros inferiores al $80 \%$ del estado inicial, pero a partir del 80\% de deterioro los efectos sobre las cuatro variables de estado anteriores se aceleraron.

En efecto, este tipo de respuesta es descrita en Church (1995), quien indica que el ancho y de la continuidad del corredor ripario se ven afectados con la regulación del río, a la vez que se producen cambios fundamentales en la continuidad el flujo y transferencia de sedimentos. También las previsiones dadas por el modelo MCD de una reducción de la vegetación riparia es verosímil, estando descrito en la bibliografía la reducción de la vegetación riparia aguas abajo de las represas (Nilsson et al., 1991; Merritt et al., 2010, Merritt and Wohl, 2002, 2006). Así, las presiones agrícolas y urbanas afectan a las márgenes fluviales y eliminan las llanuras de inundación, también generan grandes impactos en la conectividad fluvial y reducen la anchura de los paisajes ribereños naturales.

\subsubsection{Simulación de la disminución de barreras transversales}

La simulación llevada a cabo es la eliminación progresiva de barreras transversales que estaría asociada con una política de restauración fluvial de máxima intervención. Se ha simulado la desaparición secuencial por deciles de las "barreras, embalses y azudes" y en la figura 4b se muestra la respuesta de las principales variables de estado del sistema. Así, la "continuidad riparia", las "comunidades fluviales", la "vegetación riparia" y la "conectividad" mejorarían linealmente y sólo cuando se eliminase el $80 \%$ de las "barreras, embalses y azudes" transversales se produciría un fuerte incremento en la mejora de las variables ecológicas de estado.

Este resultado de la simulación MCD es coherente con lo observado, pues la fragmentación del río genera una disminución de la continuidad longitudinal y conectividad con disminución de las formaciones vegetales. Por otra parte, las barreras provocan un fuerte impacto en la migración de las especies y su diversidad (Kingsford, 2000; Jager et al., 2001). Pringle (2003) indica que la conectividad hidrológica es esencial para la integridad ecológica del paisaje y la mejora del hábitat.

\section{Conclusiones}

Los MCD han servido para caracterizar fácilmente las variables y componentes de los ecosistemas fluviales a partir de información escasa, recogida de paneles multidisciplinares de expertos. Así, en ambientes de información escasa o procedente de fuentes dispares, los MCD se han mostrado como herramientas válidas de simulación.

Los MCD pueden ser utilizados no sólo para combinar el conocimiento tácito y dispar de los expertos, sino que también puede revelar características de los componentes ecosistémicos que faciliten un análisis posterior (Tan and Özesmi, 2006). 
La participación es una parte principal de la metodología $\mathrm{MCD}$, en nuestro caso, la información aportada por 7 expertos de diferente procedencia y experiencia ha servido para proporcionar una descripción amplia del funcionamiento y utilidad del ecosistema fluvial del río Esla.

La caracterización de las componentes del sistema según FPEIR se ha realizado de manera simple mediante los indicadores "outdegrees" y "indegrees", lo que facilita la simulación del funcionamiento del sistema ante escenarios verosímiles.

Por último, los MCD pueden ser una metodología válida para el estudio de políticas de restauración fluvial y evaluación de sus efectos ecológicos y socio-económicos.

\section{Agradecimientos}

El presente trabajo es parte del proyecto REFORM, financiado por el Séptimo Programa Marco de la Unión Europea (FP7-ENV-2011-no.282656).

\section{Bibliografía}

Axelrod, R., 1976. Structure and Decision: the Cognitive Maps of Political Elites. Princeton University Press, Princeton N.J.

Bachhofer, M., Wildenberg, M., 2010. FCMappers, http://www.fcmappers.net

Banini, G.A., Bearman, R.A., 1998. Application of fuzzy cognitive maps to factors affecting slurry rheology. Int J Mineral Process, 52, 233-244.

Bougon, M., Weick, K., Binkhorst, D., 1977. Cognition in organizations: an analysis of the Utrecht Jazz Orchestra. Administrative Science Quarterly, 22, 606-639.

CEE, 2000. Directiva 2000/60/CE del Parlamento Europeo y del Consejo de 23 de octubre de 2000 por la que se establece un marco comunitario de actuación en el ámbito de la política de aguas.

CHD, 2014. Confederación Hidrográfica del Duero. http://www.chduero.es. Acceso el 22-Diciembre-2014.

Church, M., 1995. Geomorphic response to river flow regulation: Case studies and timescales. Regul River 11, 3-22.

Curia, L., Lavalle, A., 2011. Decision strategies in dynamics systems using Fuzzy Cognitive Mapping. Application to a socio-economic example. J. Inform. Syst. Technol. Manag., $8(3), 663-680$

Eden, C., Ackerman, F., Cropper, S., 1992. The analysis of cause maps. J. Manag. Studies, 29, 309-323.

González del Tánago, M., García de Jalón, D., 1995. Restauración de Ríos y Riberas. Fundación Conde del Valle de Salazar, Madrid.

Gurnell A.M., Bussettini, M., Camenen, B., Gonzalez Del Tanago, M., Grabowski, R.C., Hendriks, D., Henshaw, A., Latapie, A., Rinaldi, M., Surian N., 2014. A hierarchical multiscale framework and indicators of hydromorphological processes and forms. Deli- 
verable2.1, Part 1, of REFORM (REstoring rivers FOR effective catchment Management), a Collaborative project (large-scale integrating project) funded by the European Commission within the 7th Framework Programme under Grant Agreement 282656.

Harary, F., Norman, R.Z., Cartwright, D., 1965. Structural Models: An Introduction to the Theory of Directed Graphs. John Wiley \& Sons, New York.

Jager, H.I., Chandler, J.A., Lepla, K.B., Van Winkle, W., 2001. A theoretical study of river fragmentation by dams and its effects on white sturgeon populations. Environ. Biolog. Fish., 60, 347-361.

Kauffman, J.B., Beschta, R.L., Otting, N., Lytjen, D., 1997. An ecological perspective of Riparian and stream restoration in the Western United States. Fisheries, 22(5), 12-24.

Kingsford, R.T., 2000. Ecological impacts of dams, water diversions and river management on floodplain wetlands in Australia. Aust. Ecol., 25,109-127.

Kok, K., 2009. The potential of Fuzzy Cognitive Maps for semi-quantitative scenario development, with an example from Brazil. Global Environmental Change, 19, 122-133.

Kosko, B., 1986. Fuzzy cognitive maps. Int. J. Man-Machine Studies, 24, 65-75.

Merritt, D. M., Wohl, E. E., 2002. Processes governing hydrochory along rivers: hydraulics, hydrology, and dispersal phenology. Ecol. Appl.12, 1071-1087.

Merritt, D. M., Wohl, E. E., 2006. Plant dispersal along rivers fragmented by dams. River Res. and Appl., 22, 1-26.

Merritt, D.M., Nilsson, C., Jansson, R., 2010. Consequences of propagule dispersal and river fragmentation for riparian plant community diversity and turnover. Ecol. Monogr., 80, 609-626.

Mouratiadou, I., Moran, D., 2007. Mapping public participation in the Water Framework Difrective: a case study of the Pinios River Basin, Greece. Ecol. Econom., 62, 66-76.

Nilsson, C. et al., 1991. Long-term effects of river regulation on river margin vegetation. $J$. of Appl. Ecol., 28, 963-987.

OCDE, 1991. Environmental Indicators. A preliminary Set. OCDE, Paris, France.

Özesmi, U., Özesmi, S.L., 2003. A participatory approach to ecosystem conservation: Fuzzy Cognitive Maps and Stakeholder Group Analysis in Uluabat Lake, Turkey. Environm. Manag., 31, 518-531.

Özesmi, U., Özesmi, S.L., 2004. Ecological models based on people's knowledge: a multistep Fuzzy Cognitive Mapping approach. Ecol. Model., 176, 43-64.

Papageorgiou, E.I., Groumpos, P.P., 2005. A new hybrid method using evolutionary algorithms to train Fuzzy Cognitive Maps. Applied Soft Computing, 5, 409-431.

Papageorgiou E.I., Markinos, A.Th., Gemtos, Th., 2009. Application of fuzzy cognitive maps for cotton yield management in precision farming. Expert Systems with Applications, 36(10), 12399-12413.

Papageorgiou, E.I, Kontogianni, A., 2012. Using Fuzzy Cognitive Mapping in Environmental Decision Making and Management: A Methodological Primer and an Application, International Perspectives on Global Environmental Change. Dr. Stephen Young (Ed.), InTech.

Pringle, C., 2003. What is hydrologic connectivity and why is it ecologically important? Hydrol. Process.17, 2685-2689.

Stylios, C.D., Groumpos, P.P., 2000. Fuzzy cognitive maps in modeling supervisory control systems, Journal Of Intelligent \& Fuzzy Systems, 8, 83-98. 
Stylios, C.D., Groumpos, P.P., 2004. Modelling Fuzzy Cognitive Maps, IEEE Transactions on Systems, Man and Cybernetics Part A, 34 (1), 155-162.

Tan C.O., Özesmi, U., 2006. A generic shallow lake ecosystem model based on collective expert knowledge, Hydrobiologia, 563, 125-142. 
